\title{
The Dome: An Unexpectedly Simple Failure of Determinism
}

\author{
John D. Norton
}

Newton's equations of motion tell us that a mass at rest at the apex of a dome with the shape specified here can spontaneously move. It has been suggested that this indeterminism should be discounted since it draws on an incomplete rendering of Newtonian physics, or it is "unphysical," or it employs illicit idealizations. I analyze and reject each of these reasons.

1. Introduction. It has been widely recognized for over two decades that, contrary to the long-standing lore, Newtonian mechanics is not a deterministic theory. The clarion call came in John Earman's (1986, Chapter 3 ), which recounted the failure of determinism, including the then-recent discovery by Mather and McGehee of "space invader" systems of interacting particles that spontaneously rush into an empty space from spatial infinity. Further, simpler violations of determinism emerged. Pérez Laraudogoitia (1996) described an especially simple example of "supertask" indeterminism in which a countable infinity of masses confined to a unit interval are spontaneously energized; and Norton (1999) described a correspondingly simple example of a countable infinity of masses connected by springs that are spontaneously energized.

In this developing tradition, the simplest example so far of indeterminism in Newtonian physics is what has come to be known as "the dome," described in Norton (2003, Section 3). The indeterminism involves none of the complications of infinitely many systems interacting or masses appearing with unbounded speeds from spatial infinity. A mass sits on a dome in a gravitational field. After remaining motionless for an arbitrary

$\dagger$ To contact the author, please write to: Department of History and Philosophy of Science, University of Pittsburgh, Pittsburgh, PA 15260; e-mail: jdnorton@pitt.edu.

\$y thanks to my cosymposiasts, John Earman and David Malament; to Michael Dickson, Bernie Goldstein, Stephan Hartmann, Alexandre Korolev, and Dmitri Tymoczko; and to visiting fellows of the Center for Philosophy of Science: Boris Grozdanoff, Antigone Nounou, Hernan Pringe, and Stéphanie Ruphy.

Philosophy of Science, 75 (December 2008) pp. 786-798. 0031-8248/2008/7505-0025\$10.00 Copyright 2008 by the Philosophy of Science Association. All rights reserved. 


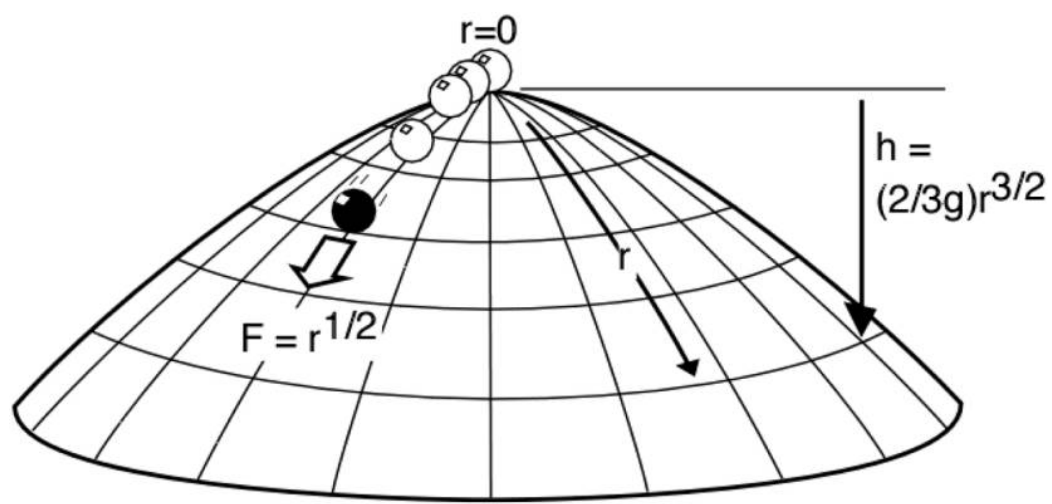

Figure 1. The dome.

time, it spontaneously moves in an arbitrary direction, with these indeterministic motions compatible with Newtonian mechanics.

This note will consider whether the dome depends on some improper maneuver in Newtonian theory. I will argue that it does not. Resolving this issue proves to be of unexpected philosophical interest. It requires a careful appraisal of three questions: Just what is Newtonian theory? What do we mean by the notion "unphysical"? Are some idealizations improper?

\section{The Dome in Brief.}

2.1. Description. The dome is a radially symmetric surface shown in Figure 1. Its shape is defined by

$$
h=(2 / 3 g) r^{3 / 2},
$$

where $r$ is the radial distance coordinate in the surface of the dome, $h$ is the vertical distance below the apex at $r=0$, and $g$ is the constant acceleration of free unit mass in the vertical gravitational field surrounding the surface. A point-like, unit mass slides frictionlessly over the surface. Initially, at time $t=0$, it is at rest exactly at the apex. The net force $F$ acting on the unit mass is directed radially outward. It is the component of the gravitational force tangent to the surface, which is $g \sin \theta$, where $\theta$ is the angle between the tangent to the surface in the radial direction and the horizontal. Since $\sin \theta=d h / d r$, we have $F=g d h / d r=r^{1 / 2}$. Newton's second law sets this force equal to the acceleration $a(t)=d^{2} r / d t^{2}$ and yields the equation of motion of the mass:

$$
d^{2} r / d t^{2}=r^{1 / 2} .
$$


The expected solution is

$$
r(t)=0,
$$

in which the mass simply remains at rest for all times $t$. Another family of solutions represents spontaneous motion at an arbitrary time $T$ in an arbitrary radial direction:

$$
r(t)= \begin{cases}(1 / 144)(t-T)^{4} & \text { for } t \geq T \\ 0 & \text { for } t \leq T .\end{cases}
$$

We see that (4) satisfies (2) if we compute the radial acceleration $a(t)=$ $d^{2} r(t) / d t^{2}$, which is

$$
a(t)= \begin{cases}(1 / 12)(t-T)^{2} & \text { for } t \geq T \\ 0 & \text { for } t \leq T,\end{cases}
$$

and note that $a(t)$ as given in (5) is the square root of $r(t)$ as given in (4).

The dome manifests indeterminism ${ }^{1}$ in the standard sense that a single past can be followed by many futures. The mass may be at rest for all times up to $t=0$. It then may or may not move spontaneously at any time after that. ${ }^{2}$

Elsewhere (Norton 2003, Section 3) I have responded to concerns about the moment $t=T$. Briefly, since no net force acts on the mass at $t=$ $T$, does Newton's first law obtain? It does, since (5) tells us the mass has zero acceleration at $t=T$. Those who yearn for a first cause to initiate the motion should recall that a requirement of a first cause is not a part of Newton's laws of motion. Further, there is no first moment of acceleration at which this first cause might act. The moment $t=T$ is the last moment of unaccelerated motion.

2.2. Individual versus Collective Indeterminism. This manifestation of indeterminism differs from those already in the literature. In the case of supertask indeterminism, each component is well behaved. If a component is set into motion, the reason is that it has been struck, pushed, or pulled by another component. The space invader form of indeterminism is odder in the sense that it involves components that pop into being "from spatial

1. Equation (2) with initial conditions $r(0)=d r(0) / d t=0$ fails to satisfy a familiar condition sufficient for existence and uniqueness of a solution, a Lipschitz condition. I originally concocted the dome example by starting with a textbook example of a violation of a Lipschitz condition and then worked backward to a plausible physical instantiation.

2. I have used the fact that Newtonian theory assigns no probabilities to these different outcomes as a way of illustrating my claim elsewhere that inductive inference need not be probabilistic (see Norton 2007, Section 8.3). 
infinity" with unbounded speeds. However, each individual component is well behaved locally. If we look at the motion of any component in some part of spacetime of finite spatial and temporal extension, the component will change its motion only if it is struck, pushed, or pulled by another component. In both supertask and space invader cases, the indeterminism arises only when we assemble these many individually well-behaved parts into the pathological totality.

The dome is unlike this. There is only one component, the mass, and no new interaction brings about its spontaneous motion. The only relevant force, the external gravitational field, acts on the mass in exactly the same way at the moment of spontaneous excitation as it had in all the moments prior. No story in terms of smaller, individually well-behaved parts is possible for why the mass changed its state of motion at just the moment of excitation and not at any other. The best we can say is that it did because it could and it could because Newton's laws allow it.

2.3. Losing Touch. As David Malament (2008) has shown, if the mass has any nonzero initial velocity at $r=0$, it flies off the dome's surface. This does not compromise the dome as an example of indeterminism in Newtonian theory; indeterminism remains in the case of zero initial velocity.

To see that losing touch arises, consider a radial motion $h(t), r(t)$ that lies on the dome's surface, with $h$ and $r$ both strictly increasing with $t$. It starts at $r=0, t=0$ with a nonzero initial velocity $v(0)=d r(0) / d t>0$. The vertical velocity is $d h / d t=d h / d r \cdot d r / d t$, where we use the fact that $h$ can also be expressed as a function of $r$, since $r$ is strictly increasing with $t$. It now follows that the vertical acceleration is

$$
d^{2} h / d t^{2}=d h / d r \cdot d^{2} r / d t^{2}+d^{2} h / d r^{2} \cdot(d r / d t)^{2} .
$$

Recalling (1), $h=(2 / 3 g) r^{3 / 2}$, we have $d h / d r=(1 / g) \cdot r^{1 / 2}$, so that

$$
d^{2} h / d r^{2}=1 /\left(2 g r^{1 / 2}\right) .
$$

Thus $d^{2} h / d r^{2}$ is infinite at the apex $h=r=0$. So, from the second term of (6), the vertical acceleration $d^{2} h / d t^{2}$ at $t=0$ must also be infinite, as long as the initial velocity $v(0)=d r(0) / d t>0$. If the mass is to remain on the surface, it must have an infinitely large vertical acceleration ${ }^{3}$ at its first instant at $t=0$. That is impossible since the greatest acceleration

3. In the case of the spontaneous motion (4), the expression (6) becomes indeterminate at $t=0$ since its second term becomes $0 / 0$. Other arguments show that the vertical acceleration $d^{2} h / d t^{2}$ at $t=T$ is zero. Combining (1) with (4), we find that $h$ varies with $(t-T)^{6}$ in the interval $t \geq T$, so that $d^{2} h / d t^{2}$ varies with $(t-T)^{4}$, which vanishes at $t=T$. 
gravity can provide is $g$, and at the apex it is $g \sin 0=0$. So the mass cannot remain on the surface.

We could modify the example slightly. We could replicate the essential properties of the dome with a rather extravagantly idealized bead on a wire. The wire of no thickness is formed into the profile of the dome and threads an infinitely small bead of unit mass that slides frictionlessly along it. The diverging of $d^{2} h / d t^{2}$ at $t=0$ shows that the wire must exert an infinite reaction force on the bead just at the moment $t=0$ if the bead is to slide along the wire from the apex with an initial velocity greater than zero. We should not dismiss momentarily infinite reaction forces. They arise in common examples in Newtonian mechanics. Consider a ball that bounces off a hard surface in which we idealize the bounce to be momentary. A momentary, infinite reaction force is needed to reverse the direction of the ball instantly.

All these difficulties disappear if we posit $a b$ initio the existence of an outward, radially directed force field in some space that exerts a force on a unit mass of magnitude $r^{1 / 2}$ in the radial coordinate $r$. It then follows automatically that the equation of motion of the unit mass is (2) and the indeterminism ensues. If the idea of a force field everywhere directed away from an empty point in space is bothersome, a slight modification can put sources at the centers of force. Imagine a source mass point that exerts a short-range, attractive force of magnitude

$$
f(s)= \begin{cases}(L-s)^{1 / 2} & \text { for } s \leq L \\ 0 & \text { for } s>L\end{cases}
$$

on a unit mass at distance $s$ from the source. If we locate the unit mass at position $r=0$ on some straight line and the attracting mass at $r=$ $L$, then the unit mass is attracted by a force of magnitude $r^{1 / 2}$ in the interval $0 \leq r<L$. Or, in Coulomb electrostatics, imagine a sphere of positive charge in three-dimensional space whose density is proportional to $1 / r^{1 / 2}$ for $r$ a radial coordinate. It turns out that the force acting on a unit test charge is outwardly directed from the singular point in the distribution at $r=0$ and is proportional to $r^{1 / 2}$.

Finally, if we just consider motions governed by the equation of motion (2), then it can be shown that, for the case of nonzero initial velocity $v(0)=d r(0) / d t>0$, there is a unique solution of the equations of motion (2). See the Appendix.

3. What's Wrong with the Dome? Many believe that the dome somehow lies outside what is proper in Newtonian theory. Three distinct bases for this judgment are described below, along with my reasons for finding them unconvincing. 
3.1. Does the Dome Employ an Incomplete Formulation of Newtonian Physics? The proposal is that Newton's three laws are an incomplete formulation of Newtonian theory. The intent of Newtonian theorists was that their theory would be deterministic and all the traditional Newtonian systems are deterministic. Therefore, any formulation of Newtonian theory that admits indeterministic motions is incomplete and must be strengthened to preclude them.

My first and final reaction to this proposal is that we must distinguish what the canonical formulation of Newtonian theory entails from what its proponents may mistakenly think their theory entails. The dome conforms to Newton's laws, so the dome is a Newtonian system. The old lore was mistaken in holding that simple systems like the mass on the dome will be deterministic in Newtonian theory.

There are two complications. First, strengthening Newton's theory is not straightforward. We need to find some additional postulate to supplement the three laws, a "fourth law." But what can it be? Merely asserting as the fourth law that determinism holds is inadequate. The three laws allow competing, indeterministic motions. That form of the fourth law is not telling us which of the competing motions, if any, are to be admitted into the theory. As we strengthen the fourth law to pick among them, we risk restricting the theory excessively and precluding systems that are unobjectionable; or we may have such a narrow formulation of the fourth law that it is tailored to resolving indeterminism only in special cases. While the proposals are preliminary, ${ }^{4}$ I have seen none yet that can resolve these problems. ${ }^{5}$

Second, it is hard to determine what Newtonian theory properly is. So it is hard to know if a proposed fourth law properly belongs in it. What Newtonian theory is cannot be established by an experiment since Newtonian theory is a false theory; and it cannot be determined by mathematical demonstration since Newtonian theory is not defined as a theorem

4. Korolev (2006) suggests that a tacit presumption of Newtonian physics is that the differential equations of motion derived from it satisfy a Lipschitz condition. A Lipschitz condition is a sufficient condition mathematically for differential equations to have a unique solution. Since it is only sufficient, it may be too strong. Tymoczko (n.d.) has proposed that the informal import of Newton's first law is not fully expressed by the condition of vanishing acceleration at an instant if the net force vanishes at that instant. Rather the law asserts in addition that this inertial motion continues over a nonvanishing time interval provided that inertial motion does not in turn trigger further forces. That leaves open the question of whether determinism is preserved if the continuing motion does trigger further forces.

5. Alper et al. (2000) in effect assert that energy and momentum conservation has been a tacit fourth law of Newtonian physics that would preclude indeterministic supertask systems from counting as properly Newtonian. 
of some grander system. Perhaps the best we can say is weak: Newtonian theory is delineated historically by the community of physicists. They have the right to add a fourth law to Newtonian theory, creating an older three-law and a newer four-law version. We should object, however, to the decree that the original three-law version is no version at all, for through most of its history, the community of Newtonians took the three laws by themselves to define the theory.

3.2. Is the Dome "Unphysical"? Another common reaction to the indeterminism of the dome is that it is "unphysical" and hence can be dismissed. This reaction may have many groundings. Perhaps most important is a sense that Newtonian theory is true, well enough, of our ordinary world and that the ordinary world just does not admit masses that spontaneously set themselves into motion.

In short, my response is that a careful examination of just what "unphysical" may mean fails to license the idea that the dome is unphysical in a way that would allow us to excise it from Newtonian physics. The principal difficulty is that the dome is intended to explore the properties of Newtonian theory, not the actual world. As a false theory, Newtonian theory can certainly have consequences that do not agree with ordinary expectations, especially if the background conditions are artfully contrived.

The sense of what is "unphysical" or "physical" is quite fundamental to the intuitions of physicists. At the same time, it is a primitive notion that has not attracted further explication. I have been able to distinguish four senses, two of which are relevant to the dome:

a) Unphysical as gauge (overdescription). For descriptive convenience, a theory may admit more structures than are in the world, such as the origin of a coordinate system in a Euclidean space. It is plainly unphysical to think of this origin as the preferred center of the space. I do not think this is the sense of unphysical at issue with the dome, since the motions described do not arise from arbitrary choices made as descriptive conveniences.

b) Unphysical as false. In this usage, a theory makes a prediction that is false and quite far from approximations to the actual. A classical electrodynamical analysis of heat radiation predicts the "ultraviolet catastrophe," that heat radiation at equilibrium has an infinite energy density. This prediction in unphysical in that it directly contradicts the finite energy densities found in experiments. The remedy is to renounce the offending theory as a false theory, or at least false in the domain in which the failed prediction was made.

If this is the sense of unphysical that is at issue with the dome, then it 
gives us no license to absolve the Newtonian theory of indeterminism. At best it would tell us that Newtonian theory is just a false theory. It is no basis for concluding that the theory does not entail the prediction.

However, we do not even have a falsified prediction. The dome is not intended to represent a real physical system. The dome is purely an idealization within Newtonian theory. On our best understanding of the world, there can be no such system. For an essential part of the setup is to locate the mass exactly at the apex of the dome and exactly at rest. Quantum mechanics assures us that cannot be done. What the dome illustrates is indeterminism within Newtonian theory in an idealized system that we do not expect to be realized in the world.

c) Unphysical as pathological. In this third case, a physical theory is used to generate conclusions that actually turn out to contradict the original theory. Of course a consistent theory cannot entail conclusions that contradict it. However, such conclusions can be deduced if we add logically incompatible assumptions to a consistent theory. The "ultraviolet catastrophe" mentioned above is an example. In electrodynamical theory, we assume that there are finite $\mathbf{E}$ and $\mathbf{B}$ fields at every point in space. We add the supposition that the radiation field has come to thermal equilibrium as described in statistical physics. We infer that, were such a state possible, it would have an infinite energy density. But that would happen only if the $\mathbf{E}$ and $\mathbf{B}$ fields diverge, contrary to the assumption of the theory.

A second illustration arises in general relativity from the incautious treatment of singularities as events in a manifold with metrical structure, whereupon they become unphysical. For at curvature singularities, the metrical structure becomes singular, in contradiction to the regular behavior presumed by the theory.

This sense is not relevant to the dome since its indeterminism does not contradict Newtonian laws.

d) Unphysical through underdescription. A theory may underdescribe or underconstrain a system's properties, so that the theory admits solutions that do not apply to the system. It is standard practice to dismiss these superfluous solutions as "unphysical." For example, consider a ball that bounces in a perfectly elastic collision with a hard floor. If its speed just before the bounce is $u$ and $v$ just after, the conservation of kinetic energy requires $u^{2}=v^{2}$. Taking square roots, we have that $u=v$ or $u=-v$. We discard the first of the two solutions as "unphysical" since it corresponds to the ball penetrating the floor without impediment.

Other, weightier examples arise in time-symmetric electrodynamical the- 
ories. They admit the time-reversed solutions in which waves collapse onto charges, energizing them. These solutions are dismissed as "unphysical" since a tacit assumption is that we are concerned only with outwardly directed radiation.

This discarding of superfluous solutions as unphysical seems to be closest to what is intended in the declaration that the dome is unphysical. For its import is that the dome can somehow be excised from Newtonian theory, without discarding the full theory. However, this fourth category cannot be applied to the dome since it requires that there is a target system we seek to describe of which we have independent knowledge. That independent knowledge is used to determine that some solutions are superfluous and may be discarded. In the case of the bouncing ball, those further conditions come from our knowledge that the floor is impenetrable, an aspect of the target system not captured in the equality $u^{2}=v^{2}$. To get physically meaningful solutions, we must implement that impenetrability by requiring that $u$ and $v$ have opposite signs.

In the case of the dome, there is no target system of which we can have independent knowledge. As we have seen, the dome cannot exist in the world. It is a creation that resides entirely within Newtonian theory. So we have no means of judging that some solutions delivered by the theory are superfluous and should be discarded.

3.3. Does the Dome Use Inadmissible Idealizations? The concern is that the idealizations required by the dome may be so extreme as to be inadmissible, so that the dome displays no inherent indeterminism in Newtonian theory, but only the pathology of an inadmissible idealization. There are many idealizations that we could identify as suspect. The mass is a point, so it has zero extension in space and infinite density. It slides frictionlessly over a perfectly even surface. The curvature of the surface diverges at the apex. ${ }^{6}$ The mass must be placed at perfect rest exactly at the apex. The surface is assumed perfectly rigid so that it does not deform under the weight of the mass. ${ }^{7}$

In short, my response will be that, while some idealizations may be inadmissible in certain circumstances, those used in the dome are admissible. Each of them can be found in one form or another in standard texts in Newtonian theory. For example, while the surface admits a curvature singularity at its apex, the tangent to the surface is everywhere defined. Hence it is more differentiable than another surface routinely

6. Malament (2008) finds this idealization no more troublesome than others routinely employed in Newtonian theory.

7. Korolev (2006) notes that allowing any elastic deformation of the surface would destroy the shape needed at the apex for indeterminism to obtain. 
appearing in Newtonian texts, a tabletop with a sharp edge formed by the intersection of two flat surfaces. At the edge, both curvature and tangent are undefined.

The complaints leveled against the idealizations of the dome seem to arise through a process of reasoning backward. The real goal is to disallow indeterminism. So when the dome manifests indeterminism, the strategy is to see if dismissal of its idealizations will defeat the indeterminism. That is simply an oblique way of imposing determinism as an extra law of Newtonian physics.

We can distinguish two types of failure of idealization, external and internal. I will argue that neither failure arises in the case of the dome.

The external failures arise when a theory tries to describe a system that is specified independently from the theory and the idealization strays too far from the target system. That form of failure is not relevant to the dome since there is no externally specified target system.

Internal failures arise when there is difficulty intrinsic to the relationship of the idealization to the theory. The strongest way this can arise is if we idealize to the extent that we contradict the theory. For example, in a theory that prohibits propagations faster than light, we cannot idealize bodies as perfectly rigid, insofar as perfect rigidity allows infinitely fast propagations through the bodies. This form of failure is not present in the dome.

A weaker internal failure may arise when idealizations are recovered as the limit of more realistic structures. A point mass is the limit of successively smaller, perfectly spherical masses of correspondingly greater density. We may be tempted to say that if some property changes discontinuously when the limit is taken, we have an inadmissible idealization. A sharp table edge is the limit of a smoothly beveled table edge with successively smaller bevels. A mass sliding off the horizontal table will be projected in a parabolic arc. As we consider successively smaller bevels, so that the limit of a sharp edge is approached, the parabolic arcs of the mass projected will approach that of the mass projected over the sharp edge. In the sequence of systems of successively smaller bevels, the property of the limit system agrees with the limit of the properties of the systems with successively smaller bevels. That commutation assures us that the idealization is benign.

Now consider domes with finite curvatures at their apexes. A point mass will remain on their apexes indefinitely, according to Newtonian theory. Thus, as the curvature become arbitrarily large, determinism will prevail. Yet when we arrive at the limit of infinite curvature, only then does indeterminism enter. The property of the limit system does not agree with the limit of the properties of the sequence of systems leading up to 
the limit system. Does this mean that the idealization of infinite curvature is inadmissible?

It does not. The failure is merely of one idealization to approximate another. That there can be discontinuous changes in the properties of systems when limits are taken is a commonplace and no reason for discounting the system approached in the limit. To give one illustration, it has long been recognized in statics that the stresses in a loaded horizontal beam supported at either end are determinate, as long as we model the beam as having some finite elasticity, no matter how small. In the limiting case, however, when the beam becomes perfectly rigid, the forces are indeterminate. We do not infer from this that the rigid case is no Newtonian structure at all. We merely allow that it is a different case with qualitatively different properties that cannot always be recovered by taking limits in elastic systems.

Elsewhere (Norton 2004, Section 2.2.3) I have described an idealized helicopter rotor. As its speed is halved and its size doubled, the nonzero lift $L$ it generates remains the same. In the limit of infinitely many doublings, we have an infinite rotor at rest that still generates lift $L$; or we would, if we mistakenly insist that its properties must agree with those arising in the limiting process.

4. Conclusion. The suggestion that the dome is "unphysical" is especially noteworthy. This notion has attracted much less attention among philosophers of science than is commensurate with its importance for physicists. For them, the notion of "physical" functions as a powerful guide and filter, with an authority that is almost as oracle-like as it is unanalyzed. In Section 3.2, I gave deflationary analysis, portraying the notion as the coalescence of four related ideas, each of which is unremarkable when examined in isolation. It will be interesting to see if further analysis bears out this deflationary view or if there is something of greater epistemic moment at hand. ${ }^{8}$

\section{Appendix: Unique Solution of the Equation of Motion}

$$
d^{2} r(t) / d t^{2}=r(t)^{1 / 2} \text { for Initial Conditions } r(0)=0, v(0)=d r(0) / d t>0
$$

Equation (2),

$$
d^{2} r / d t^{2}=r^{1 / 2}
$$

8. For recent discussion of further difficulties in establishing indeterminism in classical physics, see Wilson 2007; and for discussion of dome-like and other indeterminism in physics, see Kosyakov 2007. 
has a unique solution for the boundary condition $r(0)=0$ and $d r(0) / d t=v(0)>0$. This is established by showing that any solution $r(t)$ of (2) with these boundary conditions must satisfy the integral condition (A4) and that (A4) admits a unique solution.

If the solution satisfies $v(0)>0$ and is continuous and twice differentiable, then $v(t)$ must remain positive for some time interval following the instant $t=0$. Hence $r(t)$ is strictly increasing in $t$ in this interval, so that $v$ is a function of $r$. The demonstration of existence and uniqueness will be limited to this interval. (That is sufficient, since once the system has left $r=0$, it enters regions in which a Lipschitz condition is satisfied.)

This solution $r(t)$ must satisfy (2) rewritten as

$$
\begin{aligned}
r^{1 / 2} & =d^{2} r / d t^{2}=d v / d t=d v / d r \cdot d r / d t \\
& =v \cdot d v / d r=(1 / 2) \cdot\left(d v^{2} / d t\right),
\end{aligned}
$$

where we have used the fact that $v$ is a function of $r$. Its first integral is

$$
v^{2}(r)-v^{2}(0)=(4 / 3) r^{3 / 2},
$$

which is equivalent to

$$
v=d r / d t=\left(v^{2}(0)+(4 / 3) r^{3 / 2}\right)^{1 / 2} .
$$

Equation (A3) can be integrated to yield

$$
t=\int_{0}^{t} d t^{\prime}=\int_{0}^{r} \frac{d r^{\prime}}{\sqrt{v^{2}(0)+(4 / 3) r^{1 / 2}}}
$$

Any solution $r(t)$ must satisfy (A4) and is unique for each $v(0)$. Since the integrand of (A4) is always positive, it follows that $t$ is a unique, strictly increasing function of $r$. Hence the function $t(r)$ is invertible to the unique function $r(t)$, in which $r$ is a strictly increasing function of $t$. Therefore, also $d t / d r>0$ and $v(t)=d r / d t>0$ in the interval of the solution.

By reversing the calculations of (A1)-(A4), we can affirm that this unique $r(t)$ does solve (2). Finally, the solution cannot be more than twice differentiable at $t=0$. Differentiating (2), we have

$$
d^{3} r(t) / d t^{3}=\left(1 / 2 r(t)^{1 / 2}\right) \cdot d r / d t .
$$

At $t=0, d r / d t>0$ and $r=0$, so this third derivative diverges.

Finally, even though (2) has a unique solution with $r=0, v(0)>0$, it does not satisfy a Lipschitz condition with this boundary condition. (A Lipschitz condition is sufficient but not necessary for existence of a unique solution.) To see this, rewrite (2) as

$$
d r(t) / d t=v(t), \quad d v(t) / d t=r(t)^{1 / 2} .
$$

A Lipschitz condition is satisfied in a region surrounding the points 
$r=0, v=v(0)$ if there exists a $K$ such that for every pair of values in the region $\left(t, r_{1}, v_{1}\right)$ and $\left(t, r_{2}, v_{2}\right)$,

$$
\left(v_{1}-v_{2}\right)^{2}+\left(r_{1}^{1 / 2}-r_{2}^{1 / 2}\right)^{2} \leq K^{2}\left[\left(v_{1}-v_{2}\right)^{2}+\left(r_{1}-r_{2}\right)^{2}\right] .
$$

This can be rewritten as

$$
\left(K^{2}-1\right)\left(v_{1}^{2}-v_{2}^{2}\right)+\left[K^{2}-\frac{1}{\left(\sqrt{r_{1}}+\sqrt{r_{2}}\right)^{2}}\right]\left(r_{1}-r_{2}\right)^{2} \geq 0 .
$$

No value of $K$ can make this inequality hold since, for each $K$, its first term can be made to vanish by choosing $v_{1}=v_{2}$ and its second term can be made negative by choosing values of $r_{1}$ and $r_{2}$ close enough to zero.

\section{REFERENCES}

Alper, Joseph S., Mark Bridger, John Earman, and John D. Norton (2000), "What Is a Newtonian System? The Failure of Energy Conservation and Determinism in Supertasks", Synthese 124: 281-293.

Earman, John (1986), A Primer on Determinism. Dordrecht: Reidel.

Korolev, Alexandre (2006), "Indeterminism, Asymptotic Reasoning, and Time Irreversibility in Classical Physics", http://philsci-archive.pitt.edu/archive/00003003.

Kosyakov, Boris (2007), "Is Classical Reality Completely Deterministic?", arXiv:hep-th/ $0702185 \mathrm{v} 3$.

Malament, David (2008), “Norton's Slippery Slope”, Philosophy of Science 75 (5), in this issue.

Norton, John D. (1999), “A Quantum Mechanical Supertask”, Foundations of Physics 29: $1265-1302$.

(2003), "Causation as Folk Science", Philosophers' Imprint 3 (4), http://www .philosophersimprint.org/003004/. Reprinted in H. Price and R. Corry, Causation and the Constitution of Reality. Oxford: Oxford University Press.

(2004), "Why Thought Experiments Do Not Transcend Empiricism", in Christopher Hitchcock (ed.), Contemporary Debates in the Philosophy of Science. Oxford: Blackwell, 44-66.

(2007), "Probability Disassembled", British Journal for the Philosophy of Science 58: $141-171$

Pérez Laraudogoitia, J. (1996), “A Beautiful Supertask”, Mind 105: 81-83.

Tymoczko, Dmitri (n.d.), "Newtonian Pathologies and Newton's First Law", manuscript.

Wilson, Mark (2007), "Determinism and the Mystery of the Missing Physics", http://philsciarchive.pitt.edu/archive/00003372. 